v. Graefes Archiv für Ophthalmologie, Bd. 159, S. 29l-301 (1957)

Aus dem Laboratorium für Neuroophthalmologie (Prof. Dr. J. R. WoLTER), der Abteilung für Neuropathologie (Prof. Dr. K. ScHARENBERg) and aus der Augenabteilung (Prof. Dr. F. B. Fralick) des Universitätskrankenhauses Ann Arbor, Michigan (USA)

\title{
Der Aufbau der Nervenplexus in der Hornhaut des Schafsauges
} Von

\section{J. REIMER WoLTER *}

Mit 8 Textabbildungen

Eine Reihe von einigermaßen übereinstimmenden Berichten verschiedener Autoren haben in Ophthalmologenkreisen zu der Vorstellung geführt, daß die Verhältnisse der äußersten Nervenperipherie in der Hornhaut durch die Neuronenlehre nicht zu erklären seien. Die Endäste aller Nerven der Hornhaut sollen danach in feine, netzartige Strukturen einmünden, in denen alle Faserqualitäten zu einer geschlossenen, dreidimensionalen Einheit verschmolzen sind. Dieses Fasernetz wurde von REISER $^{7,8}$,nervöses Terminalreticulum" genannt. BoEkE1, 2 hat als ,periterminales Nervennetz" eine ähnliche Netzstruktur beschrieben, ohne daß diese beiden Autoren in Beschreibung und Deutung dieser Nervennetze je völlig übereingestimmt hätten. Das Vorkommen von umschriebenen Nervenendigungen in der Hornhaut wird von REISER abgelehnt, während BoEKE neben den Nervennetzen des Stromas Endknöpfe im Epithel beschrieben hat.

Wir haben kürzlich demonstriert, daß man in den Nervenbündeln des Hornhautstromas 3 morphologisch verschiedene Nerventypen unterscheiden kann (WoLTER ${ }^{13}$ ). Zwei dieser Fasertypen teilen sich dichotomisch und lassen sich mit ihren Endigungen in Stroma und Epithel beobachten (WoLTER ${ }^{12}$ ). Ein 3 . Typ im Stroma erwies sich als so fein, daß wir ihn bisher nur innerhalb der Nervenbündel aber nicht in seinem Endverlauf photographieren konnten (WoLTER ${ }^{13}$ ). Einen 4. Typ von Hornhautnerven mit dichotomischer Verzweigung und umschriebenen Endigungen innerhalb des Protoplasmas von Zellen konnten wir kürzlich im Hornhautendothel darstellen (WoLTER ${ }^{14}$ ). Bei diesen früheren Untersuchungen, die teils am Kaninchen und teils am Menschen durchgeführt wurden, haben wir also gefunden, daß jedenfalls 3 der vorhandenen Nerventypen sich - wie die meisten Nerven anderer Gewebe auch dichotomisch teilen und mit ihren Endästen umschriebene Endigungen formen. Von einer Verschmelzung der terminalen Fasern zu Netzstrukturen irgendwelcher Art haben wir dabei nichts gesehen.

* Mit Unterstützung des U.S. Department of Public Health, Education and Welfare, Grant No. B-475-C 3 . 
In der vorliegenden Arbeit möchten wir näher auf die Frage eingehen, ob in der Hornhaut des Auges Nervennetze vorkommen und ob diese Nervennetze tatsächlich die einzige Endformation darstellen, in die alle Nervenfasern einmünden. Weiter ist es von grundsätzlichem Interesse, ob die Nervenverhältnisse in der Hornhaut tatsächlich nicht mit der Neuronenlehre zu erklären sind. Da sich in der Hornhaut des Schafes die feinen Nervenfasern ganz besonders gut darstellen lassen, haben wir das Gewebe dieses Tieres für die vorliegende Arbeit ausgewählt. Zur Demonstration der Nerven im Endothel wurde ein Bild von der Hornhaut des Kaninchenauges herangezogen.

\section{Material und Methodik}

Sechs normale Hornhäute von den Augen dreier Schafe liegen diesen Untersuchungen zugrunde. Die Hornhäute wurden isoliert und in Bromformalin (Cajalscher Lösung) fixiert. Dann wurden dieselben auf dem Gefriermikrotom flach geschnitten. Die Schnitte wurden nach der ,panoptischen Silbercarbonatmethode“ -von Hortega versilbert, wie sie von Scharenberg und Zemax ${ }^{10}$ in der deutschen Literatur ausfübrlich beschrieben ist. Alle vorgelegten Abbildungen sind unretuchierte Mikrophotographien.

\section{Histologische Beschreibung}

Bei der Beschreibung sollen zunächst nur solche Hornhautnerven berücksichtigt werden, die sich von ihrem Eintritt in die Hornhaut am Limbus bis zu umschriebenen Endigungen einwandfrei im Mikroskop verfolgen und photographieren lassen. Dabei handelt es sich weitaus um die Mehrzahl aller Hornhautnerven.

Diese Nerven treten in Bündeln etwa in der Mitte des Stromas von allen Seiten her in die Hornhaut ein. Die Bündel enthalten dichtgelagerte Nervenfasern verschiedenen Kalibers und Schwannsche Begleitzellen (Abb. 1). Sie laufen von allen Seiten zentralwärts, teilen sich, nehmen überall Kontakt mit anderen Bündeln und werden feiner und feiner. Die Nervenfasern in den Bündeln teilen sich ebenfalls durchweg dichotomisch - in immer feinere Äste. Einzelne Fasern verlassen schon die groben Nervenbündel (Abb. l und 5). Gegen das Hornhautzentrum zu lösen sich die Bündel mehr und mehr auf und es bildet sich ein relativ dichtes Geflecht von einzelnen Nervenfasern. Unsere Abb. 2, 3, 5 und 6 zeigen Ausschnitte dieser Nervengeflechte, die in den oberflächlichen Schichten des Hornhautstromas besonders kompliziert sind.

Es ist nicht möglich, mit ein paar Mikrophotos einen vollständigen Eindruck von den relativ dichten Nervenplexus zu geben, die im Stroma dadurch entstehen, daß die einzelnen Nervenfasern sich immer weiter dichotomisch verzweigen und daß die feinen Äste einander überkreuzen. Das Bild wird dadurch noch weiter kompliziert, daß die feinen Nerven- 
äste ohne ersichtlichen Grund sehr oft - meist in ausgeprägten stumpfen oder spitzen Winkeln - umbiegen (Abb. 5 und 6). In unseren Abbildungen entsteht der Eindruck, daß die Nervenplexus nicht sehr engmaschig sind. Doch ist zu bedenken, daß die Bilder nur $10 \mu$ dicke Schnitte in der Ebene der dreidimensionalen Plexus darstellen!

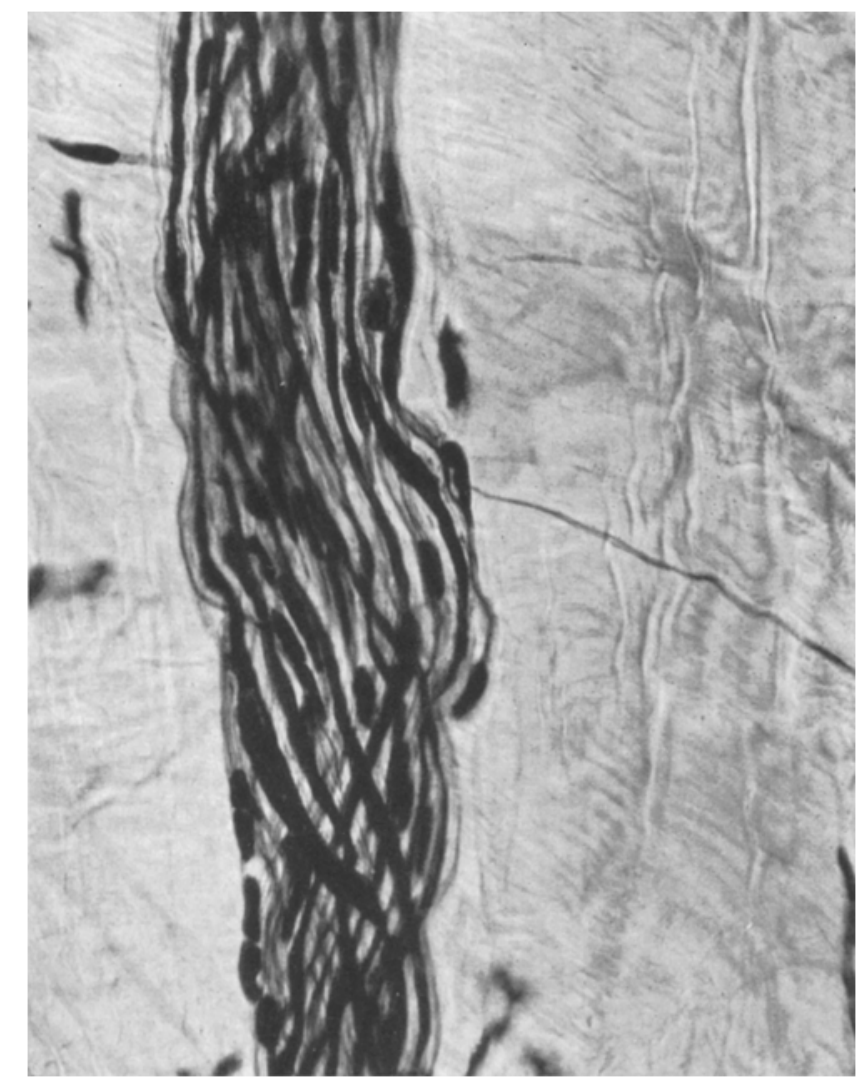

Abb. 1. Nervenbündel des peripheren Stromas der Schafshornhaut mit Nervenfasern verschiedenen Kalibers und Schwannschen Zellen. Eine Nervenfaser (a) tritt aus dem Bündel in das Stroma aus. (Hortega-Methode, Mikrophotographie, Vergr. 600x)

Beim Leser wird die Frage auftauchen, ob die hier abgebildeten Fasern auch wirklich terminale Nervenstrukturen darstellen. Daher soll besonders auf Abb. 3 hingewiesen werden, in der ein paar Nervenfasern in ihrem Verhalten zueinander abgebildet sind. Der Ast einer der Fasern formt im Bildbereich eine deutliche fußförmige Endigung an einer im Bild nur unscharf sichtbaren Stromazelle. In Abb. 4 ist der Bereich mit der Endigung bei starker Vergrößerung noch einmal besonders 


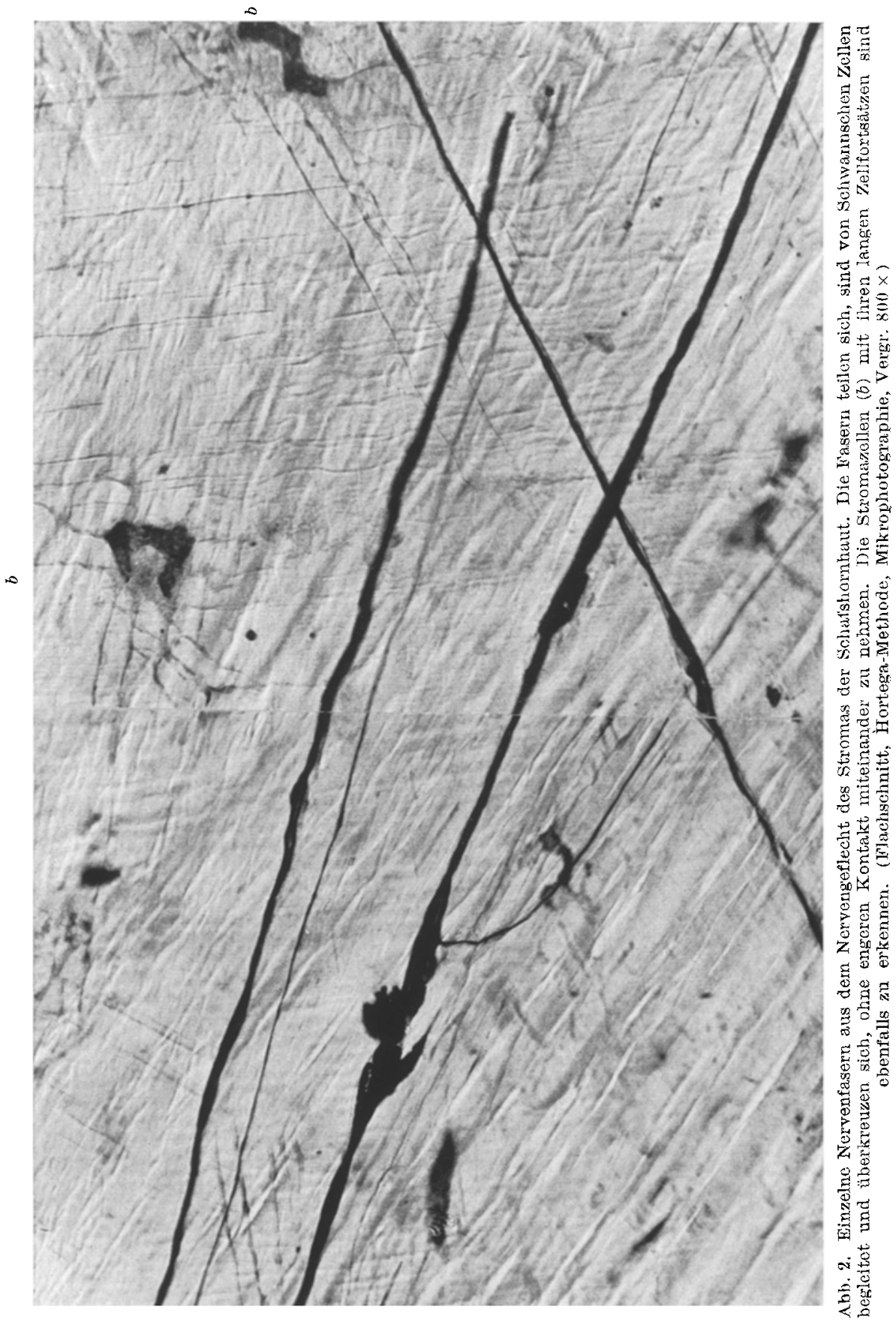


abgebildet (Pfeil). Diesen Befund sehen wir als Beweis an, da $\beta$ es sich bei den abgebildeten Fasern um ,terminale“ Nervenäste handelt.

Es läßt sich also ohne weiteres feststellen, daß die terminalen Äste solcher Nerven im Hornhautstroma zwar dichte Plexus bilden, indem sie sich verzweigen und kreuzen. Doch haben wir nicht eine einzige Stelle gefunden, an der die Nerven innerhalb dieser Plexus miteinander

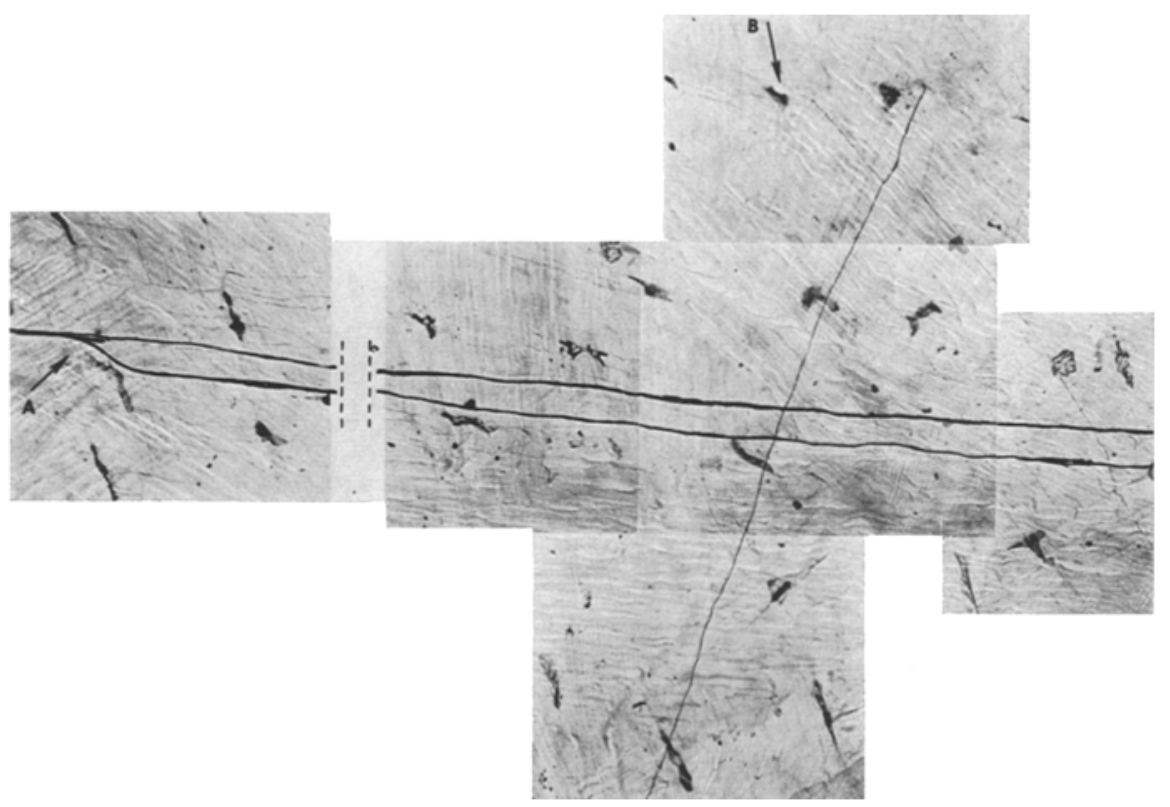

Abb. 3. Mikrophotographische Rekonstruktion von der peripheren Kreuzung von Nervenfasern im Hornhautstroma der Schafshornhaut. Eine im Bild horizontal verlaufende Faser teilt sich bei $A$ dichotomisch. Die Faser and beide Äste sind von Schwannschen Zellen begleitet. Eine zweite, feinere Faser ohne Schwannsohe Flemente verlüut im Bild vertikal und kreuzt die Äste der ersten Faser. Die vertikal verlaufende Faser entsenđet Äste ins Stroma, von denen einer bei $B$ einer Stromazelle endet. (Flachschnitt, HortegaMethode, Mikrophotographien, Vergr. $250 \times$.)

verschmolzen wären, oder in anderer Weise engen Kontakt miteinander aufgenommen hätten. Vielmehr läßt sich jede Faser - jeder Neurit als Einheit bis zu den Endigungen seiner Äste verfolgen.

Besonders dichte Fasergeflechte finden sich in den oberflächlichen Schichten des Hornhautstromas. Zahlreiche Fasern durchbrechen die Bowmansche Membran und bilden ein feines, flach angeordnetes Geflecht an dessen Außenfläche. Dasselbe ist von früheren Autoren „Plexus subepithelialis" (ConHeim) oder auch ,basales Geflecht" (BOEKE, REISER ENGELBRECHT ${ }^{3}$ ) genannt worden. Dies Geflecht entsteht wiederum, indem die Fasern, die durch die Poren der Bowmanschen Membrane 
treten, sich vielfach dichotomisch teilen (Abb. 7). Deren Äste überkreuzen sich. Doch in unseren Präparaten haben wir von einer echten Netzbildung dieser Fasern auf der Bowmanschen Membran und im basalen Epithel nichts sehen können. Vielmehr biegen die Endfasern der Nerven nach vorne um, verlassen jenes Geflecht und formen im Hornhautepithel knospenförmige Endigungen. Jedenfalls ein Teil der-

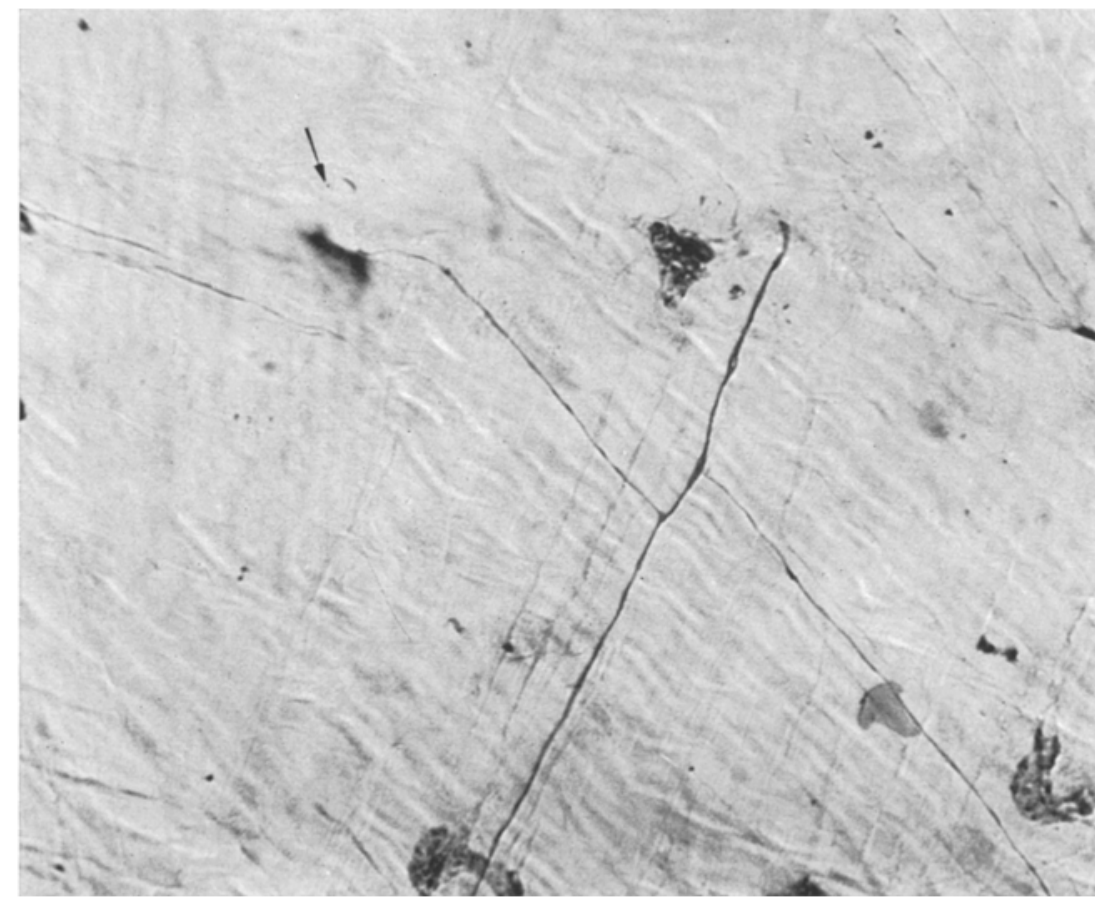

Abb. 4. Hier ist ein Teil der vertikal verlaufenden Nervenfaser aus Abb. 3 bei starker Vergrößerung dargestellt. Die Faser gibt im Bildbereich 2 Seitenäste ab. Einer derselben endet mit einem feinen kndfuß an einer nur unscharf sichtbaren Stromazelle (Pfeil). (Flachschnitt, Hortega-Methode, Mikrophotographie, Vergr. $800 \times$ )

selben liegt innerhalb des Protoplasmas von Epithelzellen. Fin Bild solcher Endfasern im Hornhautepithel des Menschen mit den knospenförmigen Endigungen haben wir kürzlich veröffentlicht und können hier darauf verweisen (WoLTER ${ }^{13}$ ).

Das Hornhautendothel wird ebenfalls von Nerven versorgt, die als grobe Fasern im Limbusbereich ins Endothel eintreten, sich dichotomisch verzweigen, feiner und feiner werden und schließlich innerhalb von Endothelzellen enden. Nerven des Endothels konnten bisher nur beim Kaninchen im Zusammenhang dargestellt werden (WoLTER ${ }^{14}$ ), während das - wahrscheinlich aus technischen Gründen - beim Menschen und 
anderen Tieren bisher nicht gelang. Abb. 8 zeigt bei schwacher Vergrößerung die sich dochotomisch verzweigenden Äste eines Endothelnerven in der Hornhaut eines normalen Kaninchens. Dieser Nerv kann bereits am Limbus im Endothel beobachtet werden. Er verläuft und teilt sich innerhalb der einschichtigen Lage von Endothelzellen, hat keine begleitenden Schwannzellen und verläuft und endet innerhalb des

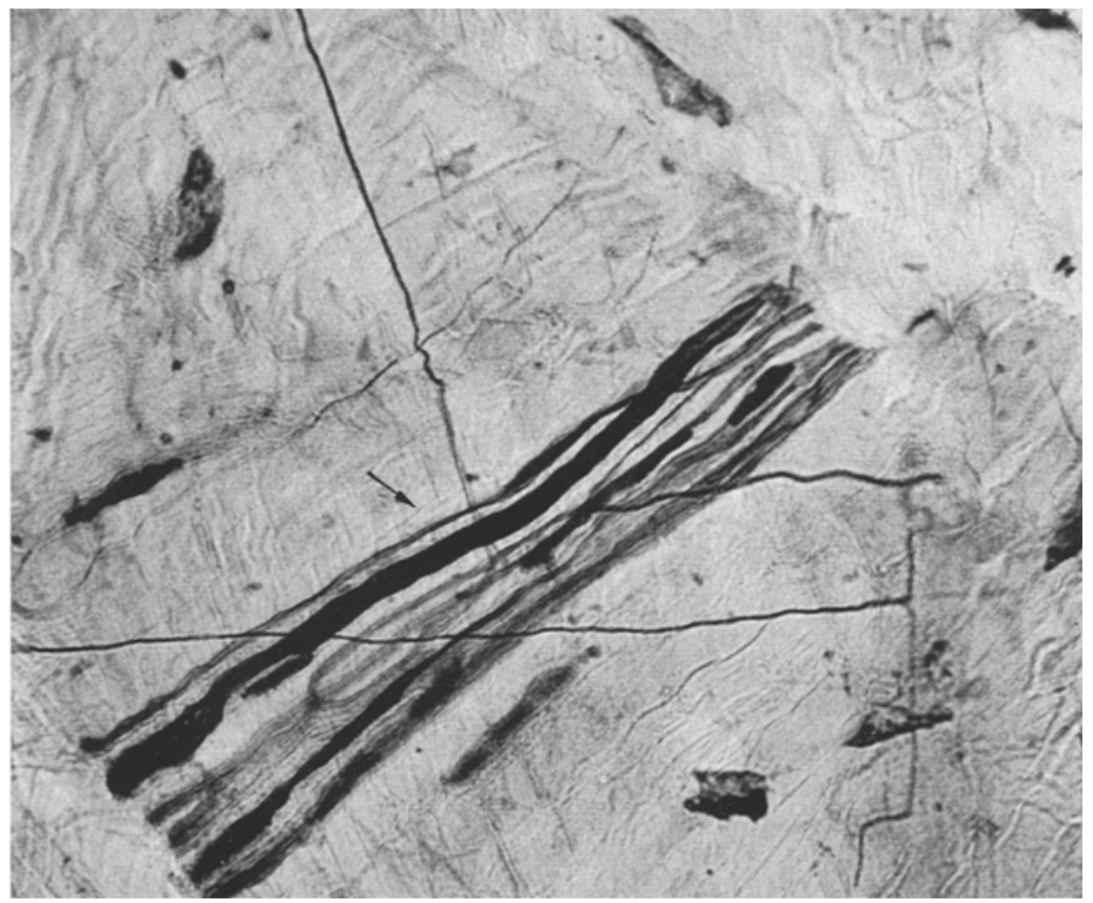

Abb. 5. Eine Nervenfaser verläBt ein Nervenbündel der normalen Schafshornhaut (Pfeil), teilt sich und verzweigt sich dann in einer Weise, die für die Nervengeflechte des Hormhantstromas typisch ist. (Flachschnitt, Hortega-Methode, Mikrophotographie, Vergr. $800 \times$ )

Protoplasmas von Endothelzellen. Von einer Netzbildung dieser Nerven kann den vorliegenden Befunden nach keine Rede sein. - Es ist wichtig hier zu bemerken, daß die Nerven des Endothels offensichtlich nicht den Bündeln der Nerven des Stromas und Epithels entstammen, die am Limbus innerhalb des Stromas in die Hornhaut eintreten. Vielmehr laufen die Endothelnerven offensichtlich einzeln durch den Bereich des Trabeculums (vg1. WoLTER ${ }^{14}$ ).

Im Epithel und im Endothel stellen Nerven mit diehotomischer Verzweigung und mit umschriebenen Endigungen in unseren Präparaten das einzig sichtbare Nervenelement dar. Im Stroma verhält sich die Mehrzahl der Nerven ebenso. Doch findet sich im Stroma außerdem ein 
ganz besonderer Nerventyp, dessen Charakteristicum darin besteht, daß derselbe überall ganz feine Netzstrukturen bildet. Diese Netze haben keine Beziehungen zu den übrigen, gröberen Nerven des Stromas. Sie stellen vielmehr ein eigenes System dar, in dem man weder Anfang noch Ende finden kann. Die Fasern dieses Typs sind im Bereich des Limbus

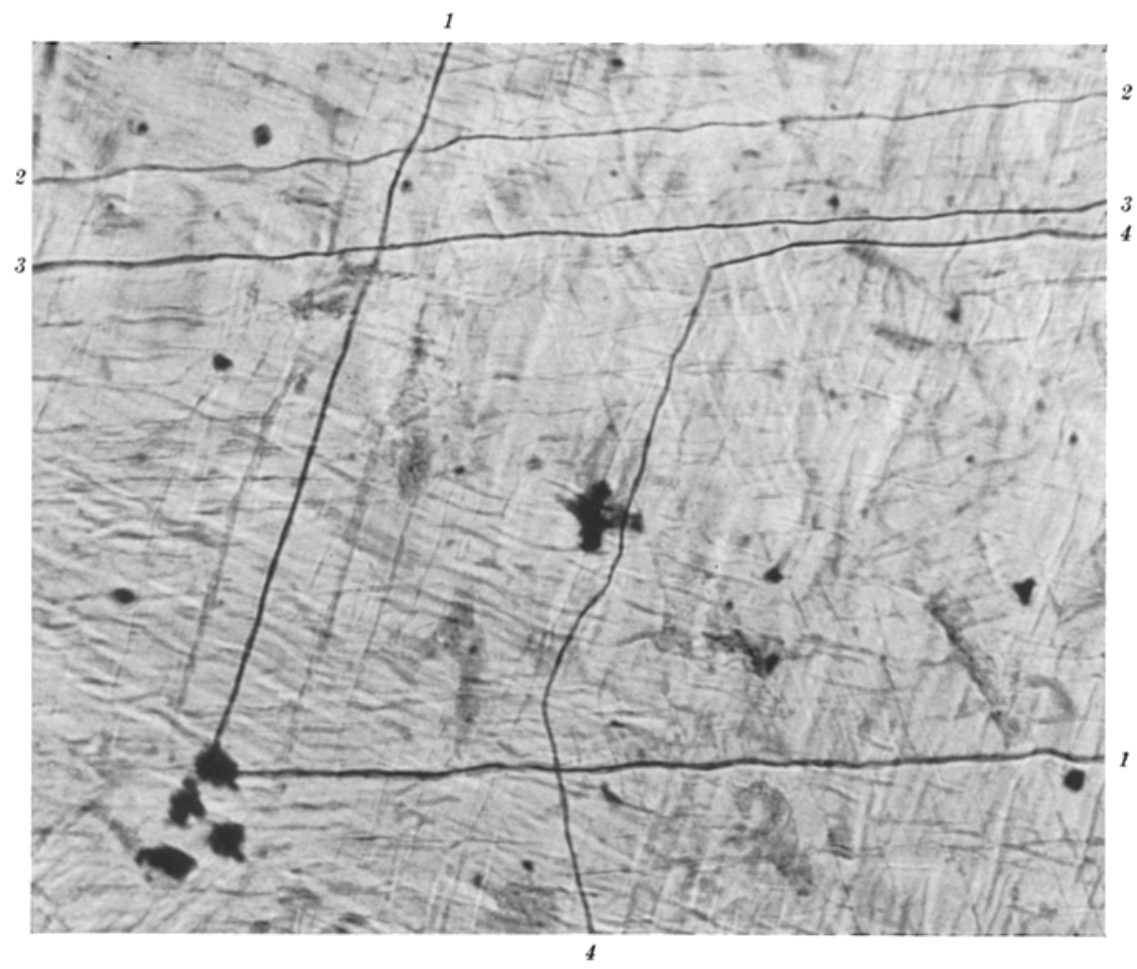

Abb. 6. Vier verschiedene feine Nervenfasem, die sich in typischer Weise überkreuzen und in stumpfen und spitzen Winkeln knicken, aus dem Stroma der Schafshornhaut. Diese Fasern gehen keine sichtbare Verbindung miteinander ein, sondern, können im Präparat teilweise zu umschriebenen Endigungen hin verfolgt werden. (Flachschnitt, Hortega-Methode, Mikrophotographie, Vergr. $900 \times$ )

nicht gröber als in der Mitte der Hornhaut und man sieht keine Endformationen. Vielmehr bilden diese Nerven netzartige Strukturen, die sich zum Hornhautzentrum hin nicht verjüngen und mit den Zellelementen des Stromas in enger Beziehung sind.

\section{Erörterung}

Der Hauptteil der Nervenfasern der Hornhaut ist also offensichtlich nichts anderes als ein dichtes System von einzelnen, weitverzweigten Neuriten. Es findet sich aber histologisch bei allen Nerven des Epithels und des Endothels und bei den meisten Nerven des Stromas kein Hinweis 
dafür, daß diese Neuriten im Bereich ihrer terminalen Strecke miteinander zu einem besonderen, neuen System verschmelzen, wie es als „,nervöses Terminalreticulum" beschrieben worden ist. Die feinen Fasern können innerhalb des Protoplasmas von Zellen verlaufen und kreuzen sich oft, sind jedoch überall deutlich voneinander trennbar. Im Epithel, im Stroma und im Endothel besitzen die Endäste dieser Neuriten um-

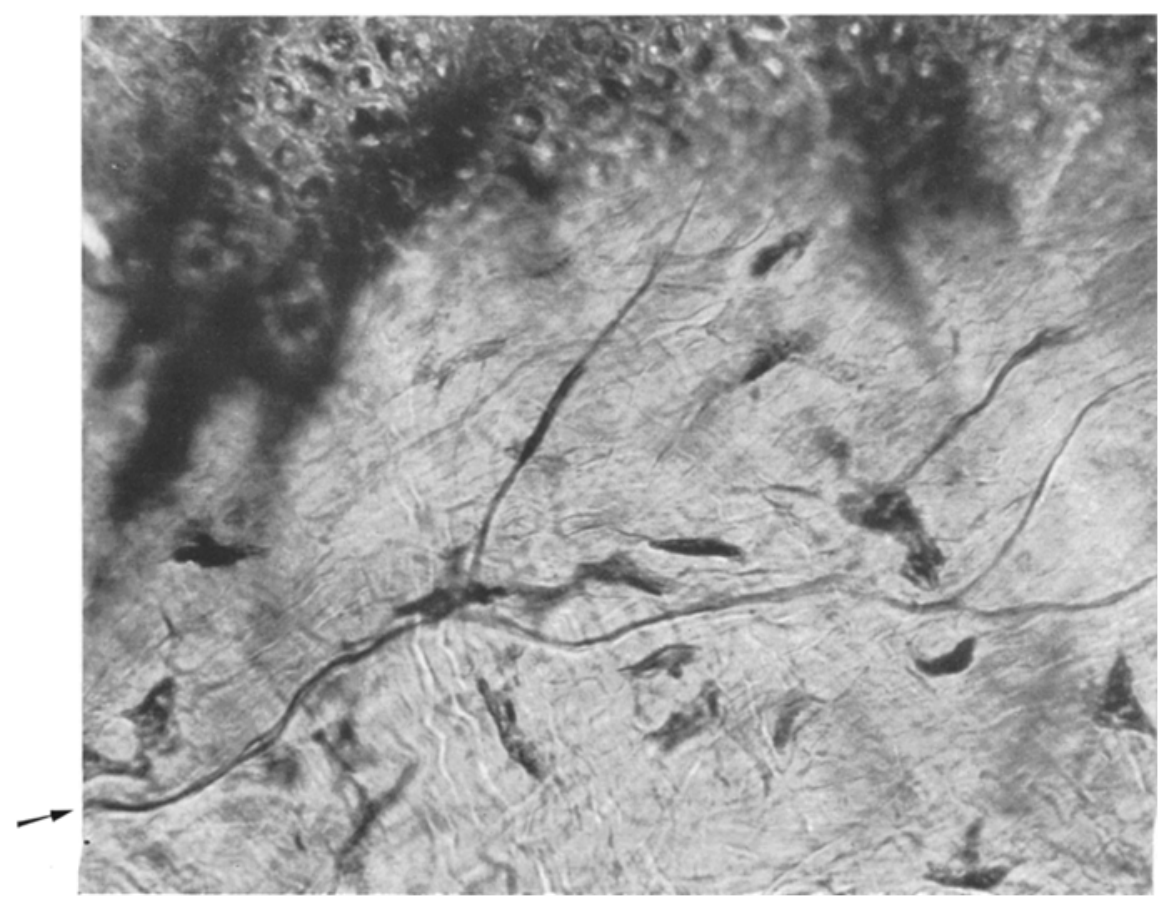

Abb. 7. Eine Nervenfaser (Pfeil), die der Bowmanschen Membran flach aufliegt und sich dichotomisch in feinste Aste teilt, aus der normalen Hornhaut des Schafes. Oben im Bild ist ein Teil des basaien Epitheis zu erkennen. (Elachschritt, Hortega-Methode, Mikrophotogtaphie, Vergr. $800 \times$ )

schriebene Nervenendigungen, die teilweise innerhalb des Protoplasmas von Hornhautzellen liegen.

Im Hornhautstroma findet sich jedoch neben zwei-früher geschilderten - verschiedenen Typen von Nerven mit dichotomischer Verzweigung und mit umschriebenen Endigungen ein besonderer 3. Nervenfasertyp. Der letztere bildet ein eigenes System von sehr feinen netzartigen Nervenstrukturen ohne erkennbare Verlaufsrichtung, das von den erstgenannten Nerven unabhängig ist. Wir haben diesen ,3. Nerventyp“ des Stromas früher beim Kaninchen beschrieben und darauf hingewiesen, daß diese netzartigen Nerven sehr an die feinen (sympathischen) Plexus 
erinnern, die die Blutgefäße anderer Gewebe begleiten (vgl. KuRUs ${ }^{4}$, WOLTER $^{11,15}$, Liss und WoLteR ${ }^{5}$ ).

Die vorliegenden Befunde bedeuten, daß die Neuronenlehre, wie sie vor über 100 Jahren bereits von CAJAL vertreten wurde, mit dem Verhalten der Mehrzahl der Nerven (Neuriten) in der Hornhaut durchaus in Einklang ist. Es erscheint uns verfrüht, darüber zu urteilen, ob das Verhalten jener feinen netzartigen Nerven tatsächlich durch die Neu-

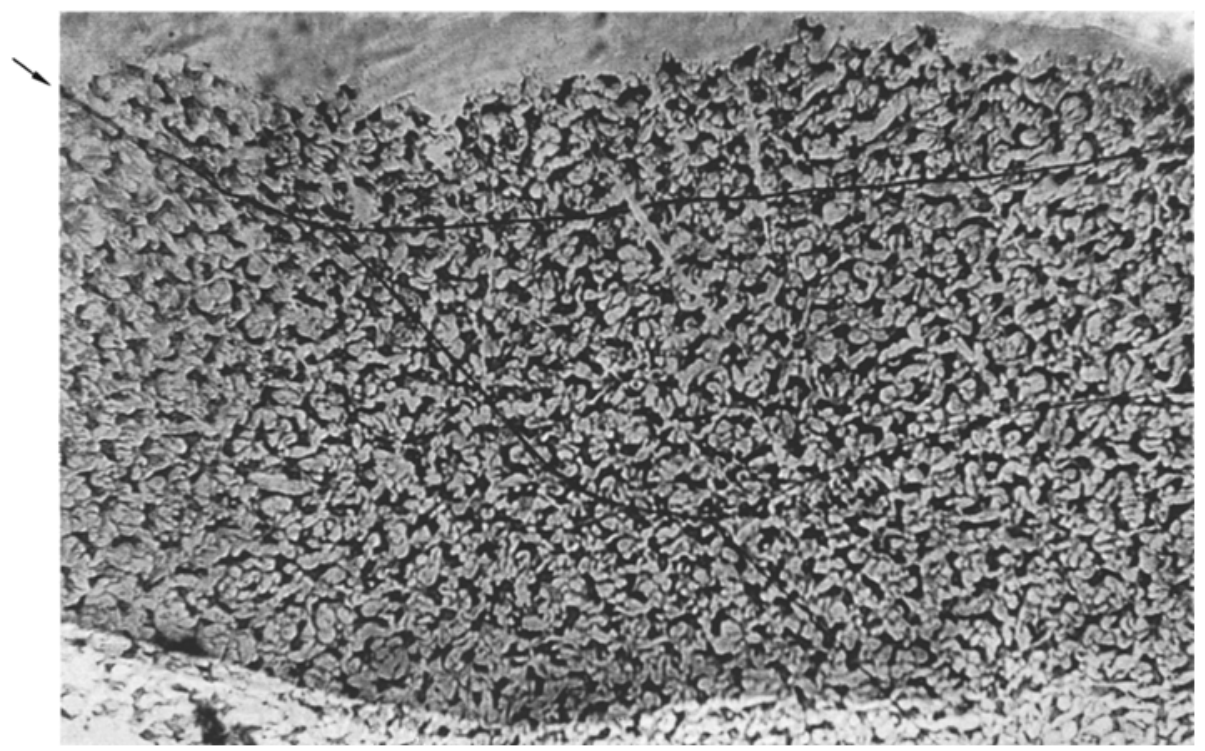

Abb. 8. Ein Nerv des Hornhautendothels des normalen Kaninohens. Der Stamm (Pfeil) teilt sich in viele Äste, die innerhalb des einschichtigen Endothels verlaufen und schließlich innerhalb von Endothelzellen enden. Dieses Bild bei schwacher Vergrößerung läßt nur die groben Äste erkennen. (Flachschnitt, Hortega-Methode, Mikrophotographie, Vergr. 250 ×)

ronenlehre nicht erklärt werden kann. Wir glauben, daß es sich bei diesem netzartigen Fasersystem um periphere Strukturen des sympathischen Nervensystem handelt. Dieselben verlaufen in anderen Geweben mit den B.utgefäßen. In der gefäßlosen Hornhaut dagegen schließen sich diese Fasernetze den Nervenbündeln an und dringen von diesen aus in das Stroma ein.

Die Existenz solcher besonderer Nerven, die ohne eigentliche Endigungen in Form eines feinen ,Endplexus" enden und teilweise innerhalb des Protoplasmas von Hornhautzellen verlaufen, ist eine Tatsache, die wir ebenso wie Boeke, Reiser, Scharenberg ${ }^{9}$, PaU und Conrads ${ }^{6}$ u. a. gesehen haben. Diese netzartigen Nerven stellen unseren Präparaten nach jedoch nur einen kleinen Teil des Nervensystems der Hornhaut dar. Den weitaus größeren Teil dagegen machen Nerven aus, die völlig 
unabhängig von den netzartigen Fasern verlaufen, sich teilen, und schließlich einfache Endigungen formen. Diese Nerven halten wir für die sensiblen und parasympathischen Elemente der Hornhaut (vgl. WoLTER $\left.{ }^{12}\right)$.

Für die vorliegende Demonstration wurden aus technischen Gründen Schnitte von der Schafshornhaut und vom Kaninchen gewählt. Eigene Untersuchungen der Hornhautnerven des Menschen ergaben grundsätzlich dieselben Befunde. Scharenderg ${ }^{9}$ hat früher bereits im Zusammenhang über die Nerven in der menschlichen Hornhaut berichtet, und festgestellt, daß darin netzförmige Nervenendstrukturen unabhängig von umschriebenen Nervenendigungen vorkommen. Letztere hält er für sensorisch. SCHARENBERgs Befunde passen also grundsätzlich zu den unseren, obgleich Scharenberg auf das hier behandelte Problem nicht näher eingegangen ist.

\section{Zusammenfassung}

Für die Existenz eines terminalen Nervennetzes, in dem alle Faserqualitäten verschmolzen sein sollen und das die alleinige Endigung aller Hornhautnerven darstellen soll, fand sich histologisch in der normalen Hornhaut des Schafes kein Hinweis.

\section{Literatur}

${ }^{1}$ Botke, J. : Z. mikrosk.-anat. Forsch. 33, 23 (1933).__2 Botke, J.: Z. mikrosk.anat. Forsch. 35 , 55 (1934). $-{ }^{3}$ ENGELBRECHT, W.: Graefes Arch. 154, 65 (1953). -${ }^{4}$ Kurus, E. : Klin. Mbl. Augenheilk. 121, 318 (1952). - ${ }^{5}$ LIss, L., u. J. R. WoLter: Klin. Mbl. Augenheilk. 129, 793 (1956). - ${ }^{6}$ PaU, H., u. H. Conrads: Graefes Arch. 157, 356 (1956). - ${ }^{7}$ Reiser, A. K.: Arch. Augenheilk. 109, 251 (1936). -${ }^{8}$ Reiser, A. K.: Arch. Augenheilk. 110, 253 (1937). - ${ }^{9}$ Scharenterg, K.: Amer. J. Ophthalm. 40, 368 (1955). - ${ }^{10}$ Scharenberg, K., u. W. Zeman: Arch. f. Psychiatr. u. Z. Neur. 188, 430 (1952). - ${ }^{11}$ WoLter, J. R.: Acta neurovegetativa (Wien) 5, 257 (1953). - ${ }^{12}$ WoLter, J. R.: Z. Zellforsch. 41, 521 (1955). - ${ }^{13}$ WoLTER, J. R.: Klin. Mbl. Augenheilk. 129, 20 (1956). - ${ }^{14}$ WoLteR, J. R.: A.M.A. Arch. Ophthalm. 58, 246 (1957). — ${ }^{15}$ WoLTER, J. R.: Graefes Arch. 158, 524 (1957).

Prof. Dr. J. Reimer Wolter, University of Michigan Hospital, Ann Arbor, USA 